\title{
A nonlinear model for rotating cool stars
}

\author{
Sydney A. Barnes ${ }^{1}$ \\ ${ }^{1}$ Lowell Observatory, 1400 W. Mars Hill Road, Flagstaff, AZ 86001, USA \\ email: barnes@lowell.edu
}

\begin{abstract}
A simple nonlinear model is introduced here to describe the rotational evolution of main sequence cool (FGKM) stars. It is formulated only in terms of the ratio of a star's rotation period, $P$, to its convective turnover timescale, $\tau$, and two dimensionless constants which are specified using solar- and open cluster data. The model explains the origin of the two sequences, $\mathrm{C} /$ fast and I/slow, of rotating stars observed in open cluster color-period diagrams, and describes their evolution from C-type to I-type through the rotational gap, g, separating them. It explains why intermediate-mass open cluster stars have the longest periods, while higher- and lower-mass cool stars have shorter periods. It provides an exact expression for the age of a rotating cool star in terms of $P$ and $\tau$, thereby generalizing gyrochronology. The possible range of initial periods is shown to contribute upto $128 \mathrm{Myr}$ to the gyro age errors of solar mass field stars. A transformation to color-period space shows how this model explains some detailed features in the color-period diagrams of open clusters, including the shapes and widths of the sequences, and the observed number density of stars across these diagrams.
\end{abstract}

Keywords. Convection, methods: analytical, stars: evolution, stars: late-type, stars: rotation

\section{Introduction}

This paper is a condensed version of Barnes (2010), which introduces a simple nonlinear model to describe the rotational evolution of cool stars, and solves it to provide an improved understanding of their spin-down. The convective turnover timescale plays a key role in this model. The theoretical context for this work can be traced to Parker (1958), Schatzman (1962), Weber \& Davis (1967), Mestel (1968), Kawaler (1988), MacGregor \& Brenner (1991), Chaboyer et al. (1995), and Noyes et al. (1984).

The immediate context for this work is provided by Barnes \& Kim (2010), who showed that the fast/C- and slow/I limits of stellar rotation, exemplified by two corresponding sequences of stars in open cluster color-period diagrams, (identified and named by Barnes 2003), can be described by

$$
\frac{d P}{d t}= \begin{cases}k_{C} P / \tau, & \text { for early times } / \text { C sequence } \\ \tau / k_{I} P, & \text { for late times } / \text { I sequence }\end{cases}
$$

where $P, t, \tau$ are, respectively, the rotation period, age, and convective turnover timescale in cool stars, and $k_{I}=452 \mathrm{Myr} / d$ and $k_{C}=0.65 d / M y r$ are two dimensionless constants. This work, described in detail in Barnes (2010), combines the two tines of that relationship into the period evolution equation,

$$
\frac{d P}{d t}=\left\{\frac{k_{I} P}{\tau}+\frac{\tau}{k_{C} P}\right\}^{-1}
$$

and summarizes its consequences for open cluster color-period diagrams. 


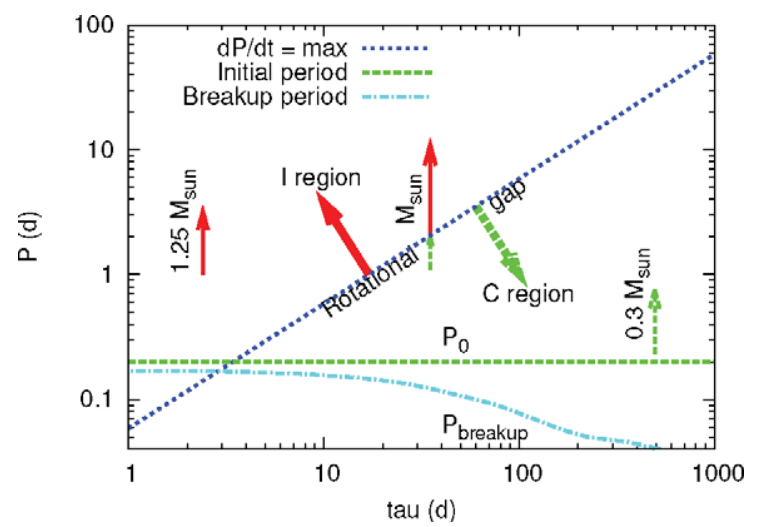

Figure 1. The $\tau-P$ plane is split into two regions $\mathrm{C} /$ (lower-right) and I/(upper left) by the straight line $P=\tau / \sqrt{k_{I} k_{C}}$ (diagonal) where $d P / d t$ is maximum. Note (1) that this line is necessarily cut by the initial period line, and (2) the locations of the individual stellar trajectories.

\section{Consequences for the $\tau-P$ plane}

$d P / d t$ approaches zero for both $P / \tau \rightarrow 0$ and $P / \tau \rightarrow \infty$, and has a maximum of $0.02 d / M y r$ when $P=\tau / \sqrt{k_{I} k_{C}}$. Consequently, as displayed in Fig. 1 , the $\tau-P$ plane is split into two regions, lower-right/C and upper-left/I by the diagonal (Rotational gap) line, where the model predicts a low number density of stars.

\section{Generalized gyro age}

Equation (1.2) can be immediately integrated to provide the gyro age, $t$, of a star:

$$
t=\frac{\tau}{k_{C}} \ln \left(\frac{P}{P_{0}}\right)+\frac{k_{I}}{2 \tau}\left(P^{2}-P_{0}^{2}\right),
$$

where $t$ is returned in Myr when $P$ and $\tau$ are specified in $d$. The gyro ages are thus generalized with respect to the original ones of Barnes (2007), where only the I-type stars were considered. Initial period variations can be shown to contribute an additional error of upto $128 \mathrm{Myr}$ for a solar-mass star.

\section{Solution in $\tau-P / P_{0}$ plane}

The solutions to the period evolution equation are conveniently displayed in the $\tau-$ $P / P_{0}$ plane (Fig. 2) (see Barnes 2010 for the $t-P$ plane), and are specified implicitly by

$$
\tau=\frac{k_{C} t \pm \sqrt{\left(k_{C} t\right)^{2}-2 k_{I} k_{C}\left(P^{2}-P_{0}^{2}\right) \ln (x)}}{2 \ln \left(P / P_{0}\right)} .
$$

Isochrones for specified ages - $100 \mathrm{Myr}$ and $1 \mathrm{Gyr}$ are displayed - have a higher-mass branch where periods increase with $\tau$, and a lower-mass branch where they decline with $\tau$. Consequently, in the context of this model, open cluster stars are expected to have a maximum rotation period at an intermediate mass, and short rotation periods for both higher- and lower-mass stars. The $100 \mathrm{Myr} \rightarrow 1 \mathrm{Gyr}$ trajectories of $1.25 M_{\odot}, 1 M_{\odot}$, and $0.3 M_{\odot}$ models are also indicated.

\section{Rotational gap}

The $\tau-P$ plane of Fig. 1 is easily transformed into the color-period plane of observations, and this transformation warps the diagonal line of maximum $d P / d t$ in the former 


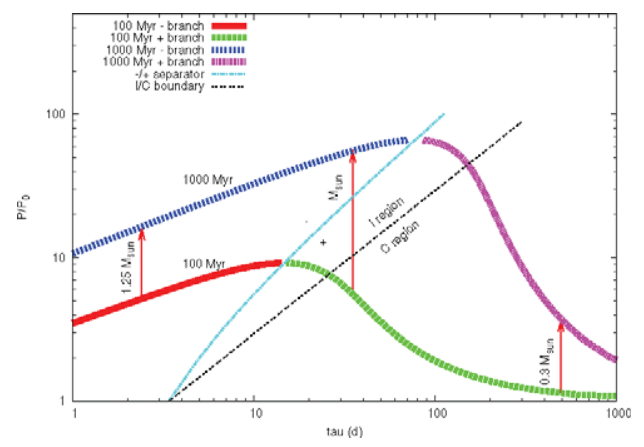

Figure 2. The solution to the period evolution equation in the $\tau-P$ plane. Note the ascent with $\tau$ of the higher-mass branch and the descent of the lower-mass branch, and the $100 \mathrm{Myr} \rightarrow 1 \mathrm{Gyr}$ trajectories for illustrative stars. (See Barnes 2010 for the dual $t-P$ plane.)

into the curved diagonal line marked in Fig. 3 for $B-V$ color. (Other colors in the set $[U B V R I J H K]$ can be obtained by using the table provided in Barnes \& Kim 2010.) As expected from the theory, we observe a remarkable paucity of stars in the vicinity of this (Rotational gap) line in both panels, the left displaying the young (100-150 Myr) Pleiades (Hartman et al. 2010) and M35 (Meibom et al. 2009) open clusters, and the right displaying the older (550 Myr) M37 open cluster (Hartman et al. 2009). The division of rotating stars into fast/C- and slow/I sequences proposed by Barnes (2003) is also clearly visible in both panels.

\section{Isochrones}

One can also calculate isochrones using an appropriate range of initial periods, and we display two panels corresponding to (1) the classic Hyades/Coma Ber (600 Myr) data of Radick et al. $(1987 ; 1990)$ and (2) the Solar datum/age.

The left panel shows the fidelity with which the position and dispersion of the observations can be accounted for, including the downturn for the coolest stars, which shows them to be of C-type. Note the absence of stars at the location of the rotational gap. The right panel shows the convergence of periods expected at solar age. It also displays
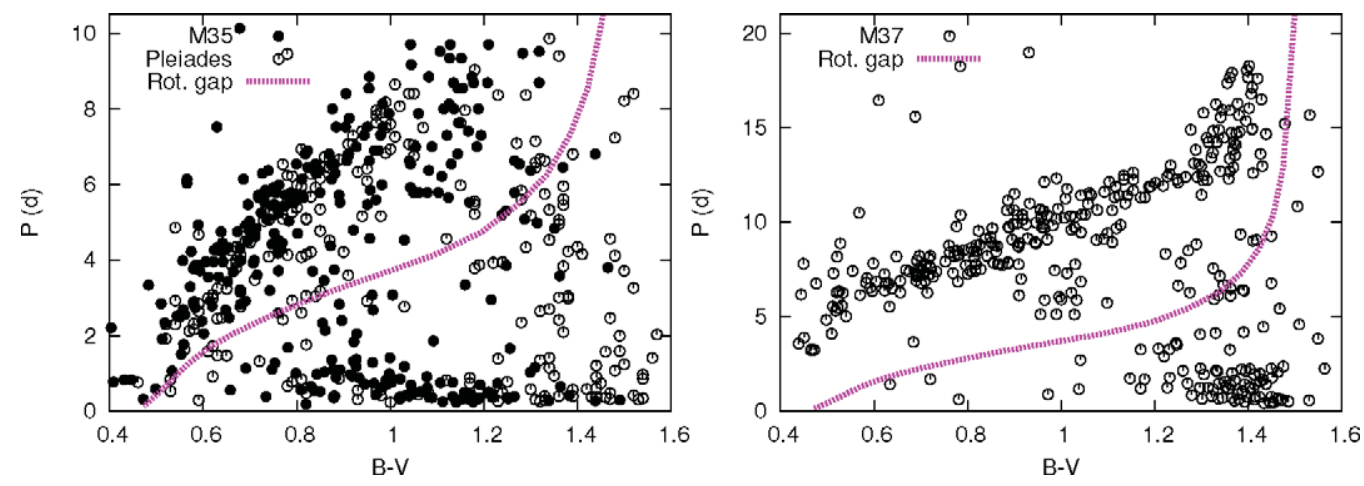

Figure 3. A paucity of stars is expected and observed at the C/I boundary (rotational gap) region (dotted pink lines) because $d P / d t$ is at a maximum here. The fast/C- and slow/I sequences proposed by Barnes (2003) are clearly visible. Left: The (100-150 Myr) Pleiades/M35 rotation period data. Right: The (550 Myr) M37 period data. 

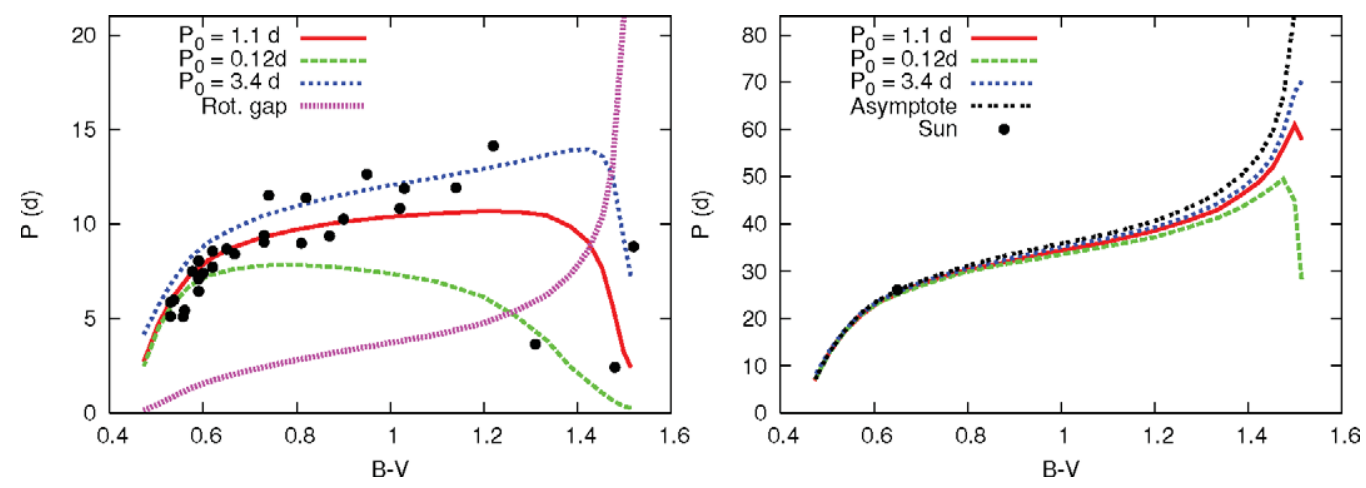

Figure 4. Left: Isochrones for $600 \mathrm{Myr}$, overlaid on the Hyades-Coma Ber rotation period data. The model reproduces the tight rotational sequence for F- and G stars, the increasing dispersion for K stars, and the fast C-type rotation for the M stars. (The dotted pink line is the calculated rotational gap.) Right: The Solar datum is overlaid on the theoretical $B-V$ Color-Period isochrones for solar age, $4.57 \mathrm{Gyr}$. Note the very tight I sequence expected for all masses but the lowest. The asymptotic relationship $P \rightarrow \sqrt{t} \times \sqrt{2 \tau / k_{I}}$ (dotted black line) is also displayed.

the asymptotic relationship $P \rightarrow \sqrt{t} \times \sqrt{2 \tau / k_{I}}$, showing the connection to the work of Skumanich (1972), and the mass dependence expected for late times.

SAB gratefully acknowledges the contributions of Y.-C. Kim of Yonsei University, Korea, to the numerics undergirding this work. The presentation of this paper at IAU Symp. 273 was possible due to partial support from the National Science Foundation grant numbers ATM 0548260, AST 0968672 and NASA - Living With a Star grant number 09-LWSTRT09-0039.

\section{References}

Barnes, S. A., 2003, ApJ, 586, 464

Barnes, S. A., 2007, ApJ, 669, 1167

Barnes, S. A., 2010, ApJ, in press

Barnes, S. A. \& Kim, Y.- C., 2010, ApJ, in press

Chaboyer, B., Demarque, P. D., \& Pinsonneault, M. H., 1995, ApJ, 441, 876

Hartman, J. D., Bakos, G. A., Kovacs, G., \& Noyes, R. W., 2010, MNRAS, in press

Hartman, J. D., Gaudi, B. S., Pinsonneault, M. H., Stanek, K. Z., Holman, M. J., McLeod, B. A., Meibom, S., Barranco, J. A., \& Kalirai, J. S., 2009, ApJ, 691, 342

Kawaler, S. D., 1988, ApJ, 333, 236

MacGregor, K. B. \& Brenner, M., 1991, ApJ, 376, 204

Meibom, S., Mathieu, R. D., \& Stassun, K. G., 2009, ApJ, 695, 679

Mestel, L., 1968, MNRAS, 138, 359

Noyes, R. W., Hartmann, L. W., Baliunas, S. L., Duncan, D. K., \&, Vaughan, A. H., 1984, ApJ, 279,763

Parker, E. N., 1958, ApJ, 128, 664

Radick, R. R., Skiff, B. A., \& Lockwood, G. W., 1990, ApJ, 353, 524

Radick, R. R., Thompson, D. T., Lockwood, G. W., Duncan, D. K., \& Baggett, W. E., 1987, ApJ, 321, 459

Schatzman, E., 1962, Annales d'Astrophysique, 25, 18

Skumanich, A., 1972, ApJ, 171, 565

Weber, E. J. \& Davis, L. L., 1967, ApJ, 148, 217 